\title{
Physical Constants as Result of the Many Hypercubic Lattices of a Multidirectional Discrete Space
}

\author{
Christiaan T. de Groot \\ Retired, University of Applied Sciences Utrecht, Netherlands \\ Email: ctdegroot@live.nl
}

How to cite this paper: de Groot, C.T. (2020) Physical Constants as Result of the Many Hypercubic Lattices of a Multidirectional Discrete Space. Journal of Modern Physics, 11, 432-447.

https://doi.org/10.4236/jmp.2020.113027

Received: February 13, 2020

Accepted: March 14, 2020

Published: March 17, 2020

Copyright ( 2020 by author(s) and Scientific Research Publishing Inc. This work is licensed under the Creative Commons Attribution International License (CC BY 4.0).

http://creativecommons.org/licenses/by/4.0/

\section{(c) (i) Open Access}

\begin{abstract}
A multidirectional discrete space consists of numerous hypercubic lattices each of which contains one of the spatial directions. In such a space, several groups of lattices can be distinguished with a certain property. Each group is determined by the number of lattices it comprises, forming the characterizing numbers of the space. Using the specific properties of a multidirectional discrete space, it is shown that some of the characterizing numbers can be associated with a physical constant. The fine structure constant appears to be equal to the ratio of two of these numbers, which offers the possibility of calculating the series of smallest numerical values of these numbers. With these values, a reasoned estimate can be made of the upper limit of the smallest distance of the discrete space of approximately the Planck length.
\end{abstract}

\section{Keywords}

Hypercubic Lattice, Multidirectional Discrete Space, Characterizing Numbers, Fine Structure Constant, Physical Constants, Planck Length

\section{Introduction}

Once in a while there is speculation about the origin of the physical constants of nature. As can be seen in the review article by Barrow [1], some authors have noticed coincidences between these constants and numbers of cosmological proportions, others see similarities between the constants and some numbers obtained in a combinatorial manner. Wesson [2] has proposed to completely eliminate the physical constants through appropriately adjusting the quantities and parameters in the fundamental equations of physics.

Here the possibility is investigated that the physical constants originate from a 
discrete space with many spatial directions. A multidirectional discrete space consists of a large but limited number of sparsely connected four-dimensional subspaces, each forming one of the spatial directions. The huge number of mutually equal subspaces offers possibilities not existing in the usual continuous space. The set of all subspaces consists of various subsets with a certain property. Because each subset consists of subspaces encompassing the entire space, the number of subspaces characterizing the subsets is timeless and valid throughout space. This property makes these characterizing numbers an ideal candidate to be related to the physical constants. For some physical constants, indeed there appears to be such a relation. This will be shown in two main steps.

In the first step to be found in Chapter 2, some required physical quantities are expressed in the units of the multidirectional discrete space. Only two units are needed, the smallest distance of a discrete space and the unit representing the diversity of subspaces in which a phenomenon is present.

In the second step of Chapter 3, it is shown that the different directions of a field in a multidirectional discrete space can be treated as independent entities. When this is combined with the results of the units' conversion, it is showed that the field in a multidirectional discrete space likely has a granular appearance. By using this possibility in combination with the many subspaces of a multidirectional space, it is shown that some of the characterizing numbers are proportional to a physical constant. In Chapter 4 it is shown, based on the huge number of subspaces, that the fine structure constant is equal to the ratio of two characterizing numbers, making the constant being an expression of the space structure. This ratio of two natural numbers, allows calculating the minimal values of the numerator and denominator from the empiric value of the fine structure constant. In Chapter 5, the minimum values of nearly all characterizing numbers are calculated in case an additional structural element of the discrete space applies.

The last chapter shows how to determine the largest numerical value of smallest distance of the discrete space with the found values of the characterizing numbers. The distance found appears to be of the same order of magnitude as the Planck length.

\section{Expressing Some Usual Physical Quantities in the Discrete Units}

A set of loosely connected hypercubic lattices (as given in [3]) is used here as an example of a multidirectional discrete space. Such a space is discrete at the level of the lattice and in the number of hypercubic lattices it consists of. The discreetness of space is expressed in the unit-distance $\Delta x(=\Delta t)$, being the minimal distance both in space and time. Due to the many lattices, an object is present in a subset of all possible lattices. To describe a particle field, an additional unit $S$ is required representing the lattices in which the object is present.

A physical quantity expressed in obvious unit $\Delta x$ and in the unusual unit $S$ is 
called a discrete quantity, indicated in the subscript: $q_{d}$. Curiously, no more units are needed. To incorporation of the physical quantities in a discrete space, the centimetre and the second need to be converted into the unit-distances $\Delta x$ in $\Delta t$. This fairly simple conversion will be described first. For that purpose it is assumed that the arithmetically obtained classical electron radius $r_{e}$, the distance from which the electron field is present, is directly proportional to the smallest size $L_{d 0}$ of the electron field in a lattice: $r_{e}=L_{d 0} \rho_{c o n}$, wherein $L_{d 0}$ is expressed in $\Delta x$.

In a lattice, the distances $\Delta x$ and $\Delta t$ are equal: $\Delta x=\Delta t$. For the sake of clarity the two units $\Delta x$ and $\Delta t$ will be indicated separately.

$$
\begin{array}{ccl}
\begin{array}{c}
\text { Name Symbol Definition } \\
\text { Conversion constant }
\end{array} & \rho_{\text {con }}=r_{e} / L_{d 0} & \mathrm{~cm} / \Delta \mathrm{x} . \\
\text { Discrete distance } & x_{d}=x / \rho_{\text {con }} & \Delta \mathrm{x} .
\end{array}
$$

The value of classical electron radius $r_{e}$ of Equation (1) is an empirical fact. $L_{d o}$ is defined in paragraph 4.3. The expectation could be that $L_{d 0}$ equals the smallest distance $\Delta x$ in a discrete space. However, in paragraph 6.2 where a method is given to determine the maximal numerical value of $L_{d o}$ it turns out to be enormous in terms of $\Delta x$. With a given $L_{d o}$ and $r_{e}$, the constant $\rho_{c o n}$ is defined.

To determine the discrete velocity, it is assumed that the maximal velocity $v_{d \max }$ runs along the space-time diagonal of each lattice, making $v_{d \max }=1$ so that:

$$
\begin{gathered}
\text { Discrete velocity } v_{d}=v / c \quad \Delta x / \Delta t(=1), \\
\text { Discrete time } t_{d}=t c / \rho_{\text {con }} \quad \Delta t .
\end{gathered}
$$

To describe the mass in a discrete space in terms of discrete units, use is made of the expression $r_{e}=e^{2} / m_{e} c^{2}$ in the CGS-system (see, for example, [4]). Subsequently, the quantities in this equation are replaced by discrete quantities, wherein the discrete speed of light equals 1 . This result in the expression is $R_{d e}=e_{d}^{2} / m_{d e}$ with which the discrete electron mass becomes:

$$
\text { Discrete electron mass } m_{d e}=e_{d}^{2} / R_{d e} S / \Delta x \text {. }
$$

The discrete elementary charge $e_{d}$ is defined in the next chapter by Equation (14). $R_{d e}$ is the size of the virtual portion of the discrete field representing its field energy as defined in paragraph 4.3. There it is shown that in a lattice $R_{d e}$ is not equal to the smallest size $L_{d o}$ of the electron field. The unit $S$ represents the lattices over which the particle field is spread.

As explained under Equation (9), the discrete and usual mass only differ by a proportionality constant making that the mass ratios are equal:

$$
m_{d p} / m_{d e}=m_{p} / m_{e}
$$

Expressed in discrete quantities, the discrete force is: $f_{d}=\Delta m_{d} v_{d} / \Delta t_{d}$ and the discrete work is the product $W_{d}=f_{d} x_{d}$. Together with (2), (3), (4) and (6), the relation between discrete work $W_{d}$ and conventional one $W$ is:

$$
\text { Discrete work } W_{d}=W m_{d e} / m_{e} c^{2} \quad S \Delta x / \Delta t^{2} \text {. }
$$


The Coulomb energy expressed in the Gaussian unit system is: $U_{c}=e^{2} / r \quad$ [4]. Transcribing of the usual quantities in this relation by the discrete ones gives the discrete potential energy of the electron:

Discrete Coulomb pot. $U_{d C}=e_{d}^{2} / r_{d} S / \Delta X$,

in which $r_{d}$ is the discrete distance between two particles. Above equation implies that the discrete elementary charge $e_{d}$ is expressed in the unit $\sqrt{S}$. Another relation for the discrete electrical potential $U_{d C}$ can be obtained by inserting the usual $U_{C}=e^{2} / r$ in the relation (7) between discrete and conventional work. Equating this relation for $U_{d C}$ with (8) gives a relation between the discrete distance $r_{d}$ and $r$. By using relation (2), these distances can be eliminated, resulting in a second expression for the conversion constant $(\Delta x=\Delta t)$ :

$$
\rho_{\text {con }}=m_{d e} / m_{e} c^{2} e^{2} / e_{d}^{2} \quad c m / \Delta x .
$$

The definition of the conversion constant $\rho_{\text {con }}$ is based on an electron. Alternatively, another charged fundamental particle could be used. Because $\rho_{\text {con }}$ is the relationship between different kind of spaces independent of the particle used to define it, it follows from (9) that the particle independence of $e / e_{d}$ also applies to $m_{d p} / m_{p}$.

According to the Gaussian unit system, the electric field strength is written as: $E=e r / r^{3}$ [4], resulting in the analogue discrete electric field strength of an electron at distance $r_{d}$ of the electron core:

$$
\text { Discrete field strength } E_{d}=e_{d} \boldsymbol{e}_{r} / r_{d}^{2} \sqrt{S} / \Delta x^{2} \text {, }
$$

with $\boldsymbol{e}_{\boldsymbol{r}}$ the unit vector between the two electrons. The discrete electric field strength will be used in the next chapter to relate $e_{d}$ with a natural number characterizing the discrete space.

Replace the quantities in the de Broglie momentum equation $m v=h / \lambda$ by the converted ones using Equations (2), (3), (6) and (9):

$$
p_{d}=m_{d} v_{d}=h c e_{d}^{2} / e^{2} \lambda_{d} S / \Delta x .
$$

By defining the discrete Planck's constant as $h_{d}=h c e_{d}^{2} / e^{2}$, the discrete de Broglie momentum relation becomes:

$$
\text { Discrete de Broglie } p_{d}=h_{d} / \lambda_{d} S / \Delta x \text {. }
$$

In $h_{d}=h c e_{d}^{2} / e^{2}$, the usual Planck's constant $\mathrm{h}$ can be replaced by the fine-structure constant $\alpha=2 \pi e^{2} / h c$ written in the Gaussian unit system:

$$
\text { Discrete Planck's const. } h_{d}=h c e_{d}^{2} / e^{2}=e_{d}^{2}(2 \pi / \alpha) S \text {. }
$$

By applying the discrete de Broglie relation to a photon, the discrete momentum of the photon becomes:

$$
\text { Discrete momentum } p_{d \text { photon }}=h_{d} / \lambda_{d \text { photon }} S / \Delta x .
$$

The conversion of the usual photon energy $W_{\text {photon }}=h c / \lambda_{d \text { photon }}$ into discrete quantities by using (2), (7), (9) and (11) gives

$$
\text { Discrete energy } W_{d \text { photon }}=h_{d} / \lambda_{d \text { photon }} S / \Delta x \text {. }
$$




\section{Discrete Physical Quantities Expressed in Characterizing Numbers}

The correspondence of physical properties with space characteristics is possible in a multidirectional discrete space, a discrete space with many spatial directions within an overarching four-dimensional space. Reference [3] gives an example of such a space where the space consists of very many hypercubic lattices, the subspaces.

A multidirectional discrete space consists of an enormous number of only sparsely interconnected subspaces, each having a different spatial direction. The set of all subspaces can be subdivided into several sets containing a number of subspaces with a specific property, each number being one of the characterizing numbers of the discrete space. These are natural numbers of limited size which are valid throughout space, such as the total number of subspaces $n_{\text {subspaces }}$ or the number of subspaces with different spatial directions $n_{\text {directions. }}$. It can easily be shown that $n_{\text {subspaces }} \gg n_{\text {directions }}$ [3]. A phenomenon placed in such a space is scattered over a subset of $n_{\text {particle }}$ subspaces.

Using of the expressions for the discrete quantities of the previous chapter, it will be demonstrated that there is a relation between the characterizing numbers and some of the physical constants. Thereby it is assumed that a field with a certain direction is only present in those subspaces with an axis in the direction of the action.

\subsection{Elementary Charge in a Multidirectional Discrete Space}

Within a tangle of subspaces, there is always a subspace that has a connecting axis through two objects. The unit vector $\boldsymbol{e}_{r}$ of Equation (10) is such a connecting axis within one of the subspaces. The electric field in one of subspaces will be used to obtain the expression of the field strength in the set of subspaces with an axis parallel to the unit vector $\boldsymbol{e}_{\boldsymbol{r}}$.

Rewrite Equation (10) to the field in one subspace. With one subspace, there is no need for the dimension $\sqrt{S}$. Further dimensional considerations indicate that the field strength in one subspace is only determined by $1 / r_{d}^{2}$. When the field strength in one lattice is as simple as possible, it is only determined by the mutual distances. The consequence is that in one subspace $e_{d}=1$, so that the field strength in one of subspaces with spatial axis $\boldsymbol{e}_{\boldsymbol{r}}$ is:

$$
E_{d \text { sub }}=\boldsymbol{e}_{r} / r_{d}^{2} \quad 1 / \Delta x^{2} .
$$

Each subspace of the discrete space consists of a three spatial axis-directions. In the set of $n_{\text {directions }}$ subspaces, each axis direction is by definition present in $n_{\text {pi- }}$ vot subspaces, a property resulting from one of the regularities in the structure of the space [3]. This means that of the $n_{\text {directions }}$ subspaces there are $n_{\text {pivot }}$ subspaces with parallel axes, from which follows that the total field in a certain direction consists of $n_{\text {pivot }}$ subspaces each of strength $\boldsymbol{e}_{r} / r_{d}^{2}$. With this, the total discrete electric field strength $\boldsymbol{E}_{d}$ in the direction $\boldsymbol{e}_{r}$ at a distance $r_{d}$ of the electron core is: 


$$
E_{d}=n_{\text {pivot }} E_{d \text { sub }}=n_{\text {pivot }} \boldsymbol{e}_{r} / r_{d}^{2} \sqrt{S} / \Delta x^{2} .
$$

Comparing this expression with (10) gives that the discrete elementary charge $e_{d}$ is equal to $n_{\text {pivot }}$. So in a discrete space, the elementary charge represents the number of parallel axes within a set of $n_{\text {directions }}$ subspaces:

$$
\text { Discrete elementary charge } e_{d}=n_{\text {pivot }} \sqrt{S} \text {. }
$$

The above equality defines the discrete elementary charge in a multidirectional discrete space assuming that $n_{\text {pivot }}$ is a known quantity (see Table 1 ).

\subsection{The Granulation of the Discrete Field}

Take an electric field in the absence of another field. To abstract from the influence of any other field, the distance $r_{d}$ in expression (13) is rewritten in $L_{d}$, being a certain variable determining the field strength in one subspace. With omission of the unit vector $\boldsymbol{e}_{\boldsymbol{n}}$ the electrical field $E_{d s u b}$ in one subspace is:

$$
E_{d s u b}=1 / L_{d}^{2} \quad 1 / \Delta x^{2} .
$$

Apparently, $L_{d}$ is a distance measure making that the field in one subspace is determined by a surface area $L_{d}^{2}$. With the above equation, the energy density $E_{d s u b}^{2}$ the field in one subspace is:

$$
E_{d \text { sub }}^{2}=1 / L_{d}^{4} \quad 1 / \Delta x^{4} .
$$

The relation for the energy density indicates that the discrete field can be divided into four-dimensional cubic-like entities of size $L_{d}^{4}$ of which the surface area $L_{d}^{2}$ forms a part. To obtain the field energy out of the energy density, a cube-shaped spatial part of the field of size $L_{d}^{3}$ is taken. To not be limited in possibilities, assume that within one subspace for unknown reasons the spatial part of the field has a repetition period in time $T_{d}$ implying that it is only partially present in time. With the gap in time, the spatial part of the field will only be present uninterrupted during the time $L_{d}$, and is not present at all in the remaining period $T_{d}$. If present, the energy density of the part of the field is $1 / L_{d}^{4}$, for the rest of the time $T_{d}$ there is no energy density at all. On average over the period $T_{d}$ the energy density is $L_{d} / T_{d}$ part of $1 / L_{d}^{4}$, making:

$$
\text { energy density }=1 / L_{d}^{3} T_{d}
$$

with this, the average discrete field energy $W_{d s u b}$ in one subspace of a spatial part of the size $L_{d}^{3}$ is equal to:

$$
W_{d s u b}=1 / T_{d} \quad 1 / \Delta t
$$

\subsection{The Field Energy of a Discrete Particle Field}

A particle field is unlimited present with decreasing strength at increasing distance to the particle core. For a particle field consisting of concentric parts, each part with size $L_{d i}$ have an associated repetition distance $T_{d i}$, where $1 / T_{d i}$ is the contribution to the energy of the field. The sum of these contributions results in the field energy $1 / T_{d \text { particle }}$ of the particle. Due to the finite nature of the total field 
energy of a particle, all various parts can be replaced by one virtual quantity $R_{d}$ particle with repetition distance $T_{d \text { particle. }}$ Being cumulative quantities, both $T_{d \text { particle }}$ and associated $R_{d \text { particle }}$ are real numbers.

The particle field is present in at least $n_{\text {directions }}$ subspaces, being the minimal number of subspaces with all possible different directions, a small subset of the set of all subspaces. With $n_{\text {directions }}$ mainly independent subspaces, the total field energy of the particle is the cumulative of the individual contribution $1 / T_{d \text { particle }}$ of each subspace ${ }^{1}$ :

$$
W_{d \text { particle }}=n_{\text {directions }} / T_{d \text { particle }} S / \Delta t .
$$

when, as usual, the total field energy is equated to the particle mass, the discrete particle mass becomes:

$$
m_{d \text { particle }}=n_{\text {directions }} / T_{d \text { particle }} S / \Delta t .
$$

\subsection{Relation Wavelength and Photon Energy}

With regard to motion in a discrete space, there is still no satisfactory description thereof, see [5] or [6]. Only in the case of the motion of a photon in a lattice, some remarks can be made based on the probable granulation of the field and its movement along the space-time diagonal.

In a multidirectional discrete space, the photon field is distributed over $n_{\text {photon }}$ subspaces, each with an axis in the direction of motion. Empirically, after annihilation of an electron-positron pair in photons, there is no remainder of the initial electron fields. In the process, the electron field within $n_{\text {directions }}$ subspaces with all possible directions is transformed into a photon field within $n_{\text {photon }}$ subspaces with an axis in the direction of motion. To make this transition possible, a subspace with an arbitrary direction is somehow connected to a subspace with a preferred axis. The space-point of the multidirectional space, being a time series of subspaces as explained in 4.1, forms such a connection. Apparently a transformation of a part of the field in the one subspace to another field in another subspace can take place via the space-point in an unknown manner. This being the case, every subspace with random axes has a unique subspace with a preferred axis, so that $n_{\text {photon }}=n_{\text {directions }}$ and

$$
W_{d \text { photon }}=n_{\text {directions }} / T_{d \text { photon }} \text {. }
$$

The time length $T_{d \text { photon }}$ is equal to the spatial wavelength of the photon: At the moment of the conversion, each part of the electron field is transformed into a part of the photon field, making that the photon must also consists of parts. The photon field is moving with $v_{\text {photon }}=1$ so its parts are present along the space-time diagonal of the subspace, meaning that the parts are moving stepwise with step size $L_{d \text { photon }}=T_{d \text { photon }}$ if the parts are contiguous.

Let there be a perfect synchronization of the presence in time of all parts of ${ }^{1}$ Note that in general a particle could be present in more, not less, than $n_{\text {directions }}$ subspaces. Is that the case, it is necessary because the discrete Planck's constant applies to all types of particles to split $n_{\text {par- }}$ ticle in subsets of $n_{\text {directions }}$ subspaces. 
the photon field. This synchronization ensures that during each period $T_{d \text { photon }}$ the parts are simultaneously present at location $z=n T_{d \text { photon }} \mid(n=$ integer $)$ in all $n_{\text {photon }}$ subspaces involved. Due to the relatively long-term presence at the location of each step, the photon had a greater chance of interaction on the spot. By the fixed mutual distances of those locations, $L_{d \text { photon }}$ can be interpreted as the discrete wavelength $\lambda_{d \text { photon }}$ of the previous chapter:

$$
W_{d \text { photon }}=n_{\text {directions }} / \lambda_{d \text { photon }} S / \Delta x \text {. }
$$

By equating this expression with the same expression for the photon energy of the last chapter (12), the discrete Planck's constant becomes:

$$
\text { Discr. Planck's constant } h_{d}=n_{\text {directions }} S \text {. }
$$

\subsection{Relation Discrete Electron Radius and Its Repetition Frequency}

Equating the two expressions (11), (16) for the discrete Planck's constant results in $n_{\text {directions }}=e_{d}^{2}(2 \pi / \alpha)$. When this relation is subsequently inserted in the mass expression (15) one gets:

$$
m_{d \text { electron }}=e_{d}^{2}(2 \pi / \alpha) / T_{d e} .
$$

The same discrete electron mass is also determined by (5): $m_{d \text { electron }}=e_{d}^{2} / R_{d e}$. $R_{d e}$ is, according to Section 3.3, the size of a virtual spatial part in which the total electron field is concentrated. In a multidirectional discrete space, the electron is thus determined by both a spatial size $R_{d e}$ and its repetition time $T_{d e}$ related by:

$$
T_{\text {de }} / R_{d e}=2 \pi / \alpha .
$$

Equation (17) will be used to relate the origin of the fine structure constant to the structure of the discrete space considered ${ }^{2}$.

\section{The Fine Structure Constant Arising from the Space Structure}

In addition to the diversity of almost independent subspaces with a spatial direction, the multidirectional discrete space has characteristics resulting from the arrangement of subspaces along the time axes. This arrangement, necessary to provide for the plurality of directions, is the cause of the fine structure constant.

The discrete space studied here consists of many four-dimensional lattices linked together over the time axis of each lattice. The subspaces differ only in the direction of the three spatial axes. The resulting number of subspaces due to this space structure is countless. This number becomes less exuberant when there are regularities in the presence of subspaces. The connection via a space-point of two subspaces at regular space-time positions in both the one as the other subspace is one of these regularities when it applies to all subspaces.

${ }^{2}$ In the case there is no time gap in the presence of a part of the field in one subspace, the discrete electron mass would be: $m_{d \text { electron }}=e_{d}^{2}(2 \pi / \alpha) / R_{d e}$. This combined with (5) results in $1=2 \pi / \alpha$, a difficult to explain result. The time gap is therefore a necessity. 
In case these regularities in space-time positions are such that the set of subspaces is limited, the space is a discrete space determined by the structural regularities. This makes the multidirectional discrete space a framework whose regularities have yet to be found. The only criteria for this are the properties of the reality and the phenomena occurring therein.

\subsection{The Space-Point of the Multiple Directional Space}

Let one of the regularities of space be such that each time axis consists of groups of $n_{\text {point }}$ directly connected vertices, each of them belonging to a lattice with a different spatial direction. After $n_{\text {point }}$ vertices, there is a connection with a vertex of the original lattice. By this way of organizing, every lattice of the space remains an ordinary hypercubic lattice with equal smallest distances in time $\Delta t$ and space $\Delta x$. A space-point is an endless series of vertices connected in time. It consists of equal parts of $n_{\text {point }}$ vertices, each of them belonging to a different lattice. The parts are repeated over time, such that after $n_{\text {point }}$ vertices the vertex belongs to the same subspace.

One of the supposed regularities of the space is that $n_{\text {point }}$ represents the same number for every space-point. Because of the succession of $n_{\text {point }}$ intermediate lattices, the smallest distance of a lattice $\Delta t$ can be subdivided into even smaller differential time steps $\delta t$ with:

$$
\Delta t=n_{\text {point }} \delta t .
$$

The space thus formed consists of parallel time axes by definition, the three spatial axes perpendicular thereto are only in exceptional cases parallel. In Figure A1 of the Appendix, a visualization is given of a limited series of coupled lattices with different spatial orientations.

\subsection{The Imbalance in the Occurrence of Subspaces}

To keep all possibilities open, presume that there is an imbalance in the number of subspaces with all possible spatial directions $n_{\text {directions }}$ in relation to the number of subspaces of each space-point: $n_{\text {directions }} \gg n_{\text {point }}$. The consequence of this imbalance is that the set of $n_{\text {directions }}$ subspaces is part of a bundle of space-points. Because all subspaces are interconnected via the space-points and the diverse lattices, many chains of space-time connections can be distinguished comprising all $n_{\text {directions }}$ subspaces. Within these chains, take the chain consisting of $t_{\text {directions }}$ consecutive equal time steps $\delta t$ that comprises all $n_{\text {directions }}$ subspaces of the bundle. Then the ratio

$$
t_{\text {directions }} / \Delta t=n_{\text {directions }} / n_{\text {point }}
$$

represents the duration of the chain in question expressed in $\Delta t$. The first and last subspaces of the chain will have three parallel spatial axes, but both subspaces cannot be the same because the ratio will generally not be an integer, necessary to be the same subspace. It will appear that above ratio is equal to $(2 \pi / \alpha)$. 


\subsection{The Fine Structure Constant and the Particle Field}

According to paragraph 3.3, the particle field in each subspace consists of parts of size $L_{d i}$ each part with a repetition distance $T_{d i}$ During the time $T_{d i}-L_{d i}$ no part is present in one subspace. Because the parts must always be present somewhere, the parts are spread in time and space over the $n_{\text {directions }}$ subspaces. The scattered parts of the particle field are contiguous, meaning that when in one subspace the presence of a part stops after the time $L_{d i}$ the presence of another part starts in a subsequent subspace. This implies that there must be a connection between the successive parts in the different subspaces in an unknown way. These connections form a chain over $n_{\text {directions }}$ consecutive subspaces with each $t$ $=L_{d i}$ a subsequent connection in another subspace. The total time distance of the chain is then $T_{d i}=L_{d i} n_{\text {directions }} / n_{\text {point }}$ expressed in $\Delta t$. To have the beginning and end of the chain in the same subspace, the size of each $L_{d i}$ is such that $T_{d i}$ is an integer. So the following relation between of two ratios of integers applies:

$$
T_{d i} / L_{d i}=n_{\text {directions }} / n_{\text {point }} .
$$

According to paragraph 3.3, particle field will consist of numerous field parts of various sizes, each part contributing $1 / T_{d i}$ to the total energy. Therewith the total energy per subspace becomes:

$$
1 / T_{d \text { particle }}=\Sigma 1 / T_{d i}=\Sigma 1 / L_{d i} n_{\text {point }} / n_{\text {directions }} .
$$

$T_{d \text { particle }}$ can also be expressed in the smallest size $L_{d 0}$ of the particle field

$$
1 / T_{d \text { particle }}=c_{p} / T_{d 0}
$$

in which $c_{p}$ is an easy to define particle dependent constant. The term $1 / T_{d \text { particle }}$ determines the discrete particle mass (15). Define the associated virtual spatial

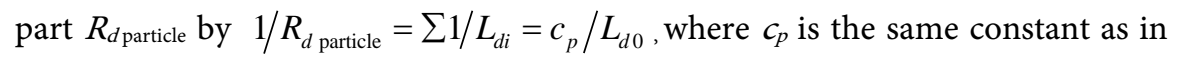
the above relation. When applied to the electron, the ratio becomes:

$$
T_{\text {de }} / R_{d e}=n_{\text {directions }} / n_{\text {point }}=T_{d 0} / L_{d 0} .
$$

The value of $L_{d 0}$ can be calculated as shown in paragraph 6.2, however, that does not apply to $R_{d e}$ which determines the discrete electron mass (5).

By combining (17) and (18), $T_{d e} / R_{d e}$ can be eliminated resulting in a particle independent relation for the fine structure constant in a discrete space:

$$
n_{\text {directions }} / n_{\text {point }}=2 \pi / \alpha \text {. }
$$

Equation (19) expresses the fine structure constant in just two characterizing numbers showing that the natural constant seems to stem from a discrete character of the space structure.

\section{Calculation of the Numerical Values of the Characterizing Numbers}

The fine structure constant $1 /(2 \pi / \alpha)$ is a well known real number. Because the fine structure constant is a ratio of two integers (19), this enables to calculate the minimal values of $n_{\text {directions }}$ and $n_{\text {point }}$.

Instead of calculating these values directly, a regularity in the arrangement of 
the subspaces of the space-point will be postulated. The chosen regularity remarkably gives the possibility to calculate almost all values of the characterizing numbers of the discrete space in the form of a broken series of natural numbers. It is used that the empirical value of $(2 \pi / \alpha)$ only is fraction larger than 861 .

\subsection{Clustering of Subspaces of the Space-Point as Regularity of the Space Structure}

Investigate the possibility that the subspaces of each space-point can be grouped into clusters of contiguous subspaces with a certain property. Each cluster covers an equal time interval:

$$
\Delta t_{\text {cluster }}=n_{\text {cluster }} \delta t \text {. }
$$

wherein $n_{\text {cluster }}$ is the number of subspaces of the cluster and $\delta t$ is the differential time step of the space-point. Let a space-point consists of $n_{\text {groupp }}$ clusters:

$$
n_{\text {point }}=n_{\text {groupp }} n_{\text {cluster }} S \text {. }
$$

Let the $n_{\text {directions }}$ subspaces with different direction also be composed by a set of $\mathrm{n}_{\text {cluster }}$ subspaces, each cluster containing mutually different spatial directions. When the set consist of $n_{\text {group }}$ clusters, the total number of subspaces is:

$$
n_{\text {directions }}=n_{\text {groupD }} n_{\text {cluster }} S \text {, }
$$

with $n_{\text {group }}>n_{\text {groupp }}$, because $n_{\text {directions }}>n_{\text {point }}$.

The last inequality means that the clusters must be present in a bundle of space-points. The space-points of the bundle do not mutually differ in the number of subspaces but in the spatial directions of the subspaces. Inserting equations (20) and (21) in relation (19) gives:

$$
2 \pi / \alpha=n_{\text {directions }} / n_{\text {point }}=n_{\text {groupD }} / n_{\text {groupP }} .
$$

\subsection{The Characterizing Number Which Determines a Cluster of Subspaces}

Let each cluster be determined by $n_{\text {spin }}$ with $n_{\text {spin }}<n_{\text {pivot }}$. $n_{\text {pivot }}$ is textually described near Equation (14). The characterizing number $n_{\text {spin }}$ stands for the number of subspaces within the cluster with a certain property. In the Appendix an example is given in which $n_{\text {spin }}$ represents the number of subspaces in the cluster with a parallel axis. In accordance with the Appendix, $n_{\text {cluster }}$ is written as a quadratic function of $n_{\text {spin }}$ with parameters $n_{c 1}$ and $n_{\mathcal{C} 2}$. These are such that $n_{\text {cluster }}$ is a natural number:

$$
n_{\text {cluster }}=n_{\text {spin }}^{2}+2 n_{c 2} n_{\text {spin }}+n_{c 1} \text {. }
$$

Due to the natural numbers that make up the characterizing numbers, equation with a ratio such as (22) can be split into two linked equations. With the extended number of equations thus obtained, nearly all characterizing numbers can be expressed in $n_{\text {cluster }}$ and $n_{\text {spin }}$ as will be shown.

\subsection{Factorizing of $(2 \pi / \alpha)$}

Use of the property that the value of $(2 \pi / \alpha)$ is very close to an integer. With this, 
$(2 \pi / \alpha)$ can be expressed in an integer value multiplied by the fraction of $n_{\text {cluster }}$ and $n_{\text {spin: }}$ :

$$
(2 \pi / \alpha)=\operatorname{Trunc}(2 \pi / \alpha) n_{\text {cluster }} / n_{\text {spin }}^{2}=\operatorname{Trunc}(2 \pi / \alpha)\left(1+2 n_{c 2} / n_{\text {spin }}+n_{c 1} / n_{\text {spin }}^{2}\right) .
$$

Use this equation and (22) to eliminate $(2 \pi / \alpha)$ :

$$
n_{\text {groupD }} / n_{\text {groupP }}=\operatorname{Trunc}(2 \pi / \alpha) n_{\text {cluster }} / n_{\text {spin }}^{2} \text {. }
$$

The above relation only consists of quantities representing an integer. The relation can therefore be split into two linked relations, one determining the numerator and the other the denominator:

$$
\begin{gathered}
n_{\text {groupD }}=\rho \operatorname{Trunc}(2 \pi / \alpha) n_{\text {cluster }}, \\
n_{\text {group }}=\rho n_{\text {spin }}^{2} .
\end{gathered}
$$

with which $n_{\text {groupD }}$ and $n_{\text {group }}$ are expressed in $n_{\text {spin }}$. The parameter $\rho$ must be such that both $n_{\text {group }}$ and $n_{\text {group }}$ are natural numbers.

More relations can be obtained by equating the expressions (11) and (16) for the discrete Planck's constant: $n_{\text {directions }}=e_{d}^{2}(2 \pi / \alpha)$. Inserting the expressions (14), (21) and (23) in this equation results in

$$
n_{\text {groupD }} n_{\text {spin }}^{2}=n_{\text {pivot }}^{2} \operatorname{Trunc}(2 \pi / \alpha),
$$

where the parameter $\rho$ is gone by equating. Together with (24), this relation gives an expression of $n_{\text {pivot }}$ in $n_{\text {spin }}$ :

$$
n_{\text {pivot }}^{2}=n_{\text {cluster }} n_{\text {spin }}^{2}
$$

\subsection{Relation Discrete Electric Charge and the Space-Point}

After combining (14), (20), (25), and (26) one obtains:

$$
\begin{gathered}
n_{\text {point }}=n_{\text {pivot }}^{2}=\rho n_{\text {spin }}^{2} n_{\text {cluster }} S, \\
e_{d}^{2}=n_{\text {pivot }}^{2}=n_{\text {point }} S .
\end{gathered}
$$

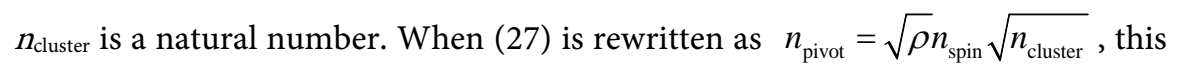
implies that $n_{\text {cluster }}$ is quadratic:

$$
\begin{aligned}
& n_{\text {cluster }}=\left(n_{\text {spin }}+n_{c 2}\right)^{2}, \\
& \text { and } n_{c 1}=n_{c 2}^{2} .
\end{aligned}
$$

Combining relation (27) with (14) and (28) gives a simple expression for the discrete electric charge $e_{d}$.

$$
e_{d}=n_{\text {pivot }}=\sqrt{\rho}\left(n_{\text {spin }}+n_{c 2}\right) n_{\text {spin }} \quad \sqrt{S} .
$$

Above relation implies that the numerical value of $\sqrt{\rho}$ is such that $\sqrt{\rho}\left(n_{\text {spin }}+n_{c 2}\right) n_{\text {spin }}$ is a natural number. Because $\rho$ must also meet the conditions formulated in the text under (25), the most simple value of $\rho=1$. This will be used for further calculations.

\subsection{The Minimum Numerical Values of the Characterizing Numbers}

The numerical value of $(2 \pi / \alpha)$ is known within a certain measurement uncertainty. 
Table 1. Some smallest characterizing numbers of discrete space.

\begin{tabular}{cccccccc}
\hline$n_{\mathcal{C} 2}$ & $n_{\text {spin }}$ & $n_{\text {cluster }}(28)$ & $n_{\text {pivot }}(29)$ & $n_{\text {point }}(27)$ & $n_{\text {directions }}(21)(24)$ & $1 / \alpha(23)$ & $\{\Delta\}$ \\
\hline 1 & 76,263 & $5.816210^{9}$ & $5.816010^{9}$ & $3.382710^{19}$ & $2.912610^{22}$ & 137.035999706 & $\{27\}$ \\
1 & 76,264 & $5.816410^{9}$ & $5.816310^{9}$ & $3.382910^{19}$ & $2.912810^{22}$ & 137.035999658 & $\{-20\}$ \\
1 & 76,265 & $5.816510^{9}$ & $5.816410^{9}$ & $3.383110^{19}$ & $2.912910^{22}$ & 137.035999611 & $\{-68\}$ \\
2 & 152,525 & $2.326410^{10}$ & $2.326410^{10}$ & $5.412210^{20}$ & $4.660010^{23}$ & 137.035999729 & $\{50\}$ \\
2 & 152,526 & $2.326510^{10}$ & $2.326510^{10}$ & $5.412410^{20}$ & $4.660210^{23}$ & 137.035999706 & $\{27\}$ \\
2 & 152,527 & $2.326510^{10}$ & $2.326510^{10}$ & $5.412510^{20}$ & $4.660310^{23}$ & 137.035999682 & $\{3\}$ \\
2 & $\ldots$ & $\ldots$ & & & & & \\
\hline
\end{tabular}

By using a search procedure, it is easy to calculate the numerical values of $n_{\text {spin }}$ out of Equation (23) via the inequality: $(2 \pi / \alpha)_{-}<=(2 \pi / \alpha)<=(2 \pi / \alpha)_{+}$. The following empirical values are used: $(2 \pi / \alpha)_{+-}=861 * 1.00002622502(69)$.

The calculation results in a series of natural numbers for $n_{\text {spin }}$ in dependence of $n_{c 2}$. Table 1 shows only the smallest values for $n_{\text {spin }}$ and, in addition, the related numerical values of some characterizing numbers. Of the numerical values shown in Table 1 are only those of $n_{\text {spin }}$ the exact values. The other values are rounded numbers whose exact values can easily be calculated with the value of $n_{\text {spin }}$ and the specified equations. To check, the last column shows the back-calculated fine structure constant using (23). For convenience: $1 / \alpha=137.035999679(94)$.

The individual values of the characterizing numbers have no error margins. The error margins can be found in the range of numerical values. Only the values of $n_{\text {spin }}$ are contiguous, the other numbers have a broken range of values.

\section{Concluding Remarks}

An old question is whether the real space is a continuous or discrete. For a long time the possibility of a discrete space was not taken seriously, mainly because of the fundamental problems with such a space such as the motion problem and the anisotropy problem [6]. With the introduction of a multidirectional hypercubic lattice seems the anisotropy problem, the problem with the many directions, to have been tackled. The multitude of equal discrete subspaces therein offers the possibility that the physical constants arise from this omnipresent abundance. In a continuous space, these constants are only difficult to explain facts.

\subsection{The Physical Constants as a Property of the Discrete Space}

The multidirectional discrete space differs from the usual Euclidean space only at the level of the smallest distance $\Delta x$. Within $\Delta x$, the space has the counterintuitive property that there are no interconnections between space-points. Also the space is unusual in the division into many sparsely connected subspaces which differ only in the spatial direction. The scarce connections enable to treat the subspaces as almost completely separate items. This has repercussions for the 
positioning of fields in a multidirectional discrete space.

The first consequence is that an additional unit is required indicating the subspaces in which the field is present. This makes it possible to convert the usual physical quantities into just two units. Secondly, the barely connected subspaces makes it very likely that the field is distributed over the various subspaces as loosely connected entities acting almost separately. This enables to express the discrete elementary charge in a number characterizing the discrete pace (14).

The implication of the units-conversion is that fields in a multidirectional discrete space should be described as a set of granulations. The graining allows expressing the discrete constant of Planck as a characterizing number of the multidirectional space (16). The consequence is that in each subspace these grains must have a gap in the presence over time, whereby the different granules form a consecutive series present in a set of subspaces. The distribution of parts of the particle field over subspaces belonging to multiple space-points can be seen as the reason for the occurrence of the fine structure constant.

\subsection{The Maximal Value of the Smallest Distance of Discrete Space}

By adding another regularity to the, as far as known, regularities in the space structure, nearly all characterizing numbers can be calculated from the fine structure constant. The diverse characterizing numbers are presented as a range of values starting with the minimal one. The size of these values depends on the measurement accuracy of the fine structure constant. The sheer size of the values found is an indication of their realism.

Not all characterizing numbers are linked here to a physical constant, such as the total number of subspaces $n_{\text {subspaces. }}$ This number must be much larger than the number of subspaces with different directions $n_{\text {directions }}=2.91 \times 10^{22} \quad$ (Table 1 ), indicating that the physical constant associated with $n_{\text {subspaces }}$ will be very tiny. The gravitational constant is good candidate.

To obtain an indication of the smallest size $L_{d o}$ of a particle field, ratio (18) is combined with (22):

$$
T_{d 0} / L_{d 0}=n_{\text {directions }} / n_{\text {point }}=n_{\text {group }} / n_{\text {group } \mathrm{P}} .
$$

Above relation indicates that various smallest sizes $L_{d 0}$ are possible, depending on the various ways the fraction $n_{\text {directions }} / n_{\text {point }}$ can be expressed in smaller numbers by reduction using the numerical values of $n_{\text {directions }}$ and $n_{\text {point }}$. When each thus obtained $L_{d o}$ belongs to a fundamental particle, a series of such particles is possible varying from particles with relatively light mass for large $L_{d 0}$ to heavier particles with a small $L_{d 0}$.

The smallest size $L_{d o}$ of discrete electron field is needed to determine the conversion constant $\rho_{c o n}$. The electron probably has the relatively large value

$L_{d 0}=n_{\text {point }}$, enabling heavier particles with smaller $L_{d 0}$. With

$n_{\text {point }}=3.3827 \times 10^{19}$ from Table 1 and the empirical electron radius

$r_{e}=2.82 \times 10^{-15} \mathrm{~m}$, the conversion constant $\rho_{c o n}$ can be calculated with (1). This 
$\rho_{\text {con }}$ inserted in (2) gives as the largest possible value of the unit-distance of the lattice:

$$
\Delta x=8.33 \times 10^{-35} \mathrm{~m},
$$

based on the electron radius $L_{d 0}=3.38 \times 10^{19} \Delta x$.

$\Delta x$ is in accordance with the Planck length $L_{P}=K_{l} 4.05 \times 10^{-35} \mathrm{~m}$, with $K_{l}$ a dimensionless constant, as obtained by dimensional analysis (see Meschini [7]). This is remarkable because the method to obtain $\Delta x$ only depends on the value of the fine structure constant and a model of electron radius $L_{d 0}$, without the use of the gravitational constant.

Expressed in $\Delta x$, the size of the discrete electron radius $L_{d o}$ is enormous. This implies that within $L_{d o}$ the field is uniformly constant, because there are no smaller parts of the field than $L_{d o}$. Therewith, the problem with the electron radius is not solved, but shifted to the problem of describing fields in a discrete space.

\section{Conflicts of Interest}

The author declares no conflicts of interest regarding the publication of this paper.

\section{References}

[1] Barrow, J.D. (1981) Quarterly Journal of the Royal Astronomical Society, 22, 388-420.

[2] Wesson, P.S. (1992) Space Science Reviews, 59, 365-406. https://doi.org/10.1007/BF00242090

[3] De Groot, C.T. (2017) Journal of Modern Physics, 8, 1175-1189. http://www.scirp.org/journal/JMP/ https://doi.org/10.4236/jmp.2017.88078

[4] Landau, L. and Lifshitz, E. (1971) The Classical Theory of Fields. Oxford: Pergamon Press, 90, 91, 88.

[5] van Bendegem, J.P. (1995) Logique et Analyse, 38, 127-150.

[6] Forrest, P. (1995) Synthese, 103, 337, 339. https://doi.org/10.1007/BF01089732

[7] Meschini, D. (2007) Foundations of Science, 12, 277-294.

https://doi.org/10.1007/s10699-006-9102-3 


\section{Appendix A. A Possible Clustering of Subspaces}

Take the possibility that the subspaces of a space-point are clustered into groups of subspaces with a spatial axis in a specific direction. The direction differs for each cluster. Within each cluster there are $n \|$ subspaces with mutual parallel spatial axes, the other axes of these subspaces have arbitrary directions. All clusters have an equal number of $n_{\text {cluster }}$ subspaces.

Take as parameter the number of subspaces in a cluster with a parallel axis: $n_{\text {spin. }}$. Let these subspaces be evenly spread over the time axis with an mutual time distance proportional to $n_{\text {spin: }}: n_{\text {spin }}+n_{c}$ (see Figure A1). So

number of subspaces with parallel axes: $n \|=n_{\text {spin }}$,

time difference of the consecutive parallel axes: $\Delta t \|=n_{\text {spin }}+n_{c}$, time interval to the next cluster $\Delta t_{c}$, gives as the number of subspaces of a cluster:

$$
n_{\text {cluster }}=\Delta t_{\text {cluster }}=n\|\cdot \Delta t\|+\Delta t_{c}=n_{\text {spin }}^{2}+n_{c} n_{\text {spin }}+\Delta t_{c}
$$

Depicted a series of time connected vertices, each belonging to different subspaces, forming a small part of the time-axis of a space-point. A cluster is formed by $n_{\text {cluster }}$ vertices in which there are $n \|$ parallel spatial axes positioned at regular time interval $\Delta t||$. The other spatial axes are random. Also in other clusters are subspaces with an axis parallel to $\|$. Only within the cluster the parallel axes are at regular distances in the time.

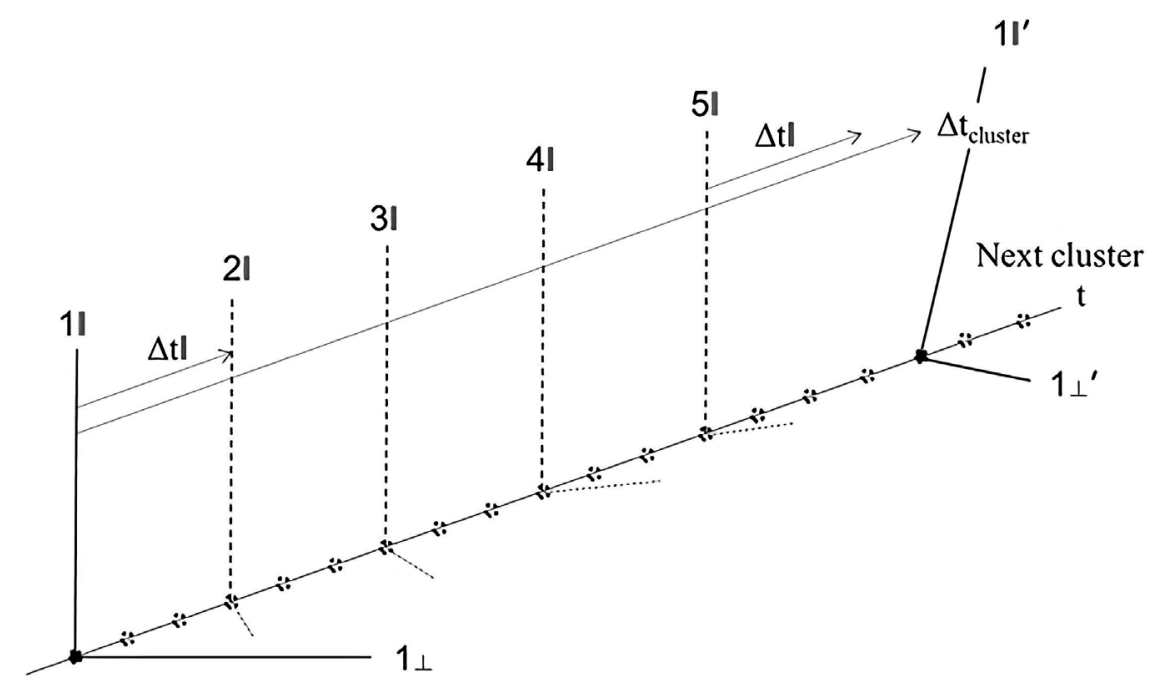

Figure A1. Clustering of subspaces. 